\title{
Minkowski units in certain metacyclic fields
}

by

\section{RoMAN MARSZaleK (Wrocław)}

1. Let $K$ be a normal extension of the rationals with metacyclic Galois group of order $p m$, where $p$ is a prime and $m \mid p-1$. The main result of this paper (Theorem 2) yields certain necessary conditions for $K$ to have a Minkowski unit; in case of prime $m$ and small $p$ they are also sufficient (Theorem 2 (iii)). The first of these conditions (conditon (b)) is a relation involving the class numbers of certain subfields of $K$, condition (c) requires the surjectivity of the norm from the units in $K$ to those in the only normal proper subfield of $K$, and condition (d) requires the existence of a Minkowski unit in the above-mentioned subfield.

Our results are similar to those obtained by D. Duval ([2], Théorème 5.2) concerning real fields with Galois groups of the type $(p, p)$. We shall also correct a mistake in the papers of N. Moser [5] and [6] (Lemme VI.5 and Lemme 4.4, respectively).

We shall use the following notation and definitions:

For any number field $\Lambda$ we put:

$U_{\Lambda}=$ the group of units in $\Lambda$,

$V_{A}=$ the group of roots of unity in $\Lambda$,

$E_{A}=$ the quotient group $U_{A} / V_{A}$.

If $A$ is normal, then the above abelian groups have a natural structure of $Z[\operatorname{Gal}(\Lambda / Q)]$-modules. For any group $\Gamma$ acting on a set $X$, we define:

$$
\begin{aligned}
& X^{r}=\{x \in X: \gamma x=x, \gamma \in \Gamma\}, \\
& \Gamma^{\sim}=\sum_{\gamma \in \Gamma^{+}} \gamma \in Z[\Gamma],
\end{aligned}
$$

$\langle\gamma\rangle=$ the subgroup of $\Gamma$ generated by $\gamma \in \Gamma$.

We say that a normal extension $\Lambda$ of the rationals with $\Gamma=\mathrm{Gal}(\Lambda / Q)$ has a Minkowski unit if $E_{A}$ is a cyclic $Z[\Gamma]$-module. In the case of real $\Lambda$ this holds if and only if $\Lambda$ has a conjugate system of fundamental units.

Let $M_{1}, M_{2}$ be $Z[I]$-modules and let $f$ be a 1-cocycle from 
$Z^{1}\left(\Gamma, \operatorname{Hom}_{Z}\left(M_{2}, M_{1}\right)\right)$. Recall that $f$ is al map $\Gamma \rightarrow \operatorname{Hom}_{Z}\left(M_{2}, M_{1}\right)$ such that if we write $f(\gamma)(m)=f_{\gamma}(m)$, then for all $m \in M_{2}$ and $\gamma, \delta \in \Gamma$ we have

$$
f_{\gamma \delta}(m)=\gamma f_{\delta}(m)+f_{\gamma}(\delta m) \text {. }
$$

On the $Z$-direct sum $M_{1} \oplus M_{2}$ we can define the action of $\Gamma$ by

$$
\gamma\left(m_{1}, m_{2}\right)=\left(\gamma m_{1}+f_{\gamma}\left(m_{2}\right), \gamma m_{2}\right)
$$

for $\gamma \in \Gamma$ and $m_{1} \in M_{1}, m_{2} \in M_{2}$. The $Z[\Gamma]$-module $M$ obtained in this way is denoted by $\left(M_{1}, M_{2} ; f\right)$. If the choice of the cocycle $f$ is obvious we write simply $M=\left(M_{1}, M_{2}\right)$. The $Z[\Gamma]$-module $M$ is called an extension of $M_{1}$ by $M_{2}$.

2. Let $G$ be a metacyclic group of order $p m$, where $p$ is a prime and $m \mid p-1$. Thus

$$
G=\left\{\sigma^{i} \tau^{j}: i=0, \ldots, p-1 ; j=0, \ldots, m-1\right\}
$$

and $\tau \sigma=\sigma^{r} \tau$, where $r$ is an $m$ th primitive root of unity $\bmod p$.

Let $K$ be a normal extension of the rationals with $\operatorname{Gal}(K / Q)=G$. We put:

$k=K^{\langle\sigma\rangle}$,

$L=K^{\langle\tau\rangle}$,

$\zeta$ is a $p$ th primitive root of unity,

$A$ is the ring of integers in $Q(\zeta)$,

$\mathscr{P}=A(1-\zeta)$,

$\psi$ is the automorphism of $Q(\zeta)$ mapping $\zeta$ onto $\zeta$,

$A_{1}=A \cap Q(\zeta)^{\langle\psi\rangle}$,

$\mathscr{P}_{1}=A_{1} \cap \mathscr{P}$

$\theta$ is an $m$ th primitive root of unity.

On $A$ and $Z[0]$ we can introduce a $Z[G]$-module structure in the following way. We let $\sigma$ act on $A$ as multiplication by $\zeta$, and $\tau$ as the automorphism $\psi$. On $\boldsymbol{Z}[\theta], \sigma$ acts trivially and $\tau$ acts as multiplication by 0 . In this way all ideals of $\boldsymbol{Z}[0]$ as well as those of $A$ which are $\psi$-invariant acquire a $Z[G]$-module structure.

Consider the $Z[G]$-modules

$$
\widetilde{E}_{K}=\left\{x \in E_{K}: x^{1+\sigma+\ldots+\sigma^{p-1}}=1\right\} \text { and } E_{0}=\widetilde{E}_{K} / E_{K} .
$$

Thus $E_{\mathrm{K}}$ is a $Z[G]$-extension of $\widetilde{E}_{K}$ by $E_{0}$. It was shown in [5] (Prop. III6) that

$$
E_{K} \simeq \bigoplus_{j=0}^{m-1} \mathscr{P e _ { j }} \mathfrak{a}_{j}
$$

where $0 \leqslant e_{j} \leqslant m-1$ and the $\mathfrak{a}_{j}$ are ideals of $A_{1}$, the class of the ideal $\prod_{j=0}^{m-1} a_{j}$ being uniquely determined by $E_{K}$. Since $\sigma$ acts trivially on $E_{0}$ one obtains

$$
E_{K} \simeq\left(\bigoplus_{j=0}^{m-1} \mathscr{P}^{e_{j}} \mathfrak{a}_{j}, \mathfrak{b}\right)
$$

where $\mathfrak{b}$ is a $Z[\langle\tau\rangle]$-module of $Z$-rank $m-1$.

If we put

$$
a=\left(E_{K^{\prime}}: E_{L} E_{L^{\sigma}} \ldots E_{l^{\mathrm{m} m} \cdot 1} E_{k}\right)
$$

then by Théorème 7 of [3] we get the equality

$$
h_{K} p^{(m-1)(m+2) / 2}=a h_{k} h_{L}^{m} .
$$

3. We now give a necessary condition for $K$ to have a Minkowski unit in the general case:

Theorem 1. If a real metacyclic extension $K$ of degree pm over $Q$ has a Minkowski unit, then

$$
h_{k} h_{L}^{m}=p^{t} h_{K} \quad \text { with } t \geqslant m-1 .
$$

Proof. Using the formula $\tau^{j} \sigma^{i}=\sigma^{i r^{j}} \tau^{j}$ and Corollaire to Proposition I3 of [7], we have

$$
E_{\sigma^{j w}(L)}=E_{K}^{\left\langle\sigma^{j_{\tau}}\right\rangle}=\left(E_{K}\right)^{\left\langle\sigma^{\left.j_{\tau}\right\rangle}\right.} \quad \text { and } \quad E_{k}=\left(E_{K}\right)^{\langle\sigma\rangle}
$$

where $w(r-1) \equiv 1(\bmod p), 0 \leqslant j \leqslant m-1$. Since $E_{K} \simeq R=Z[G] / Z G^{\sim}$, by Proposition I3 of [7], we get

$$
\left(E_{K}: E_{L} E_{\sigma^{w}(L)} \ldots E_{\sigma^{w(m-1)}(L)} E_{k}\right)=\left(R: \sum_{j=0}^{m-1} R^{\left\langle\sigma^{j} \tau\right\rangle}+R^{\langle\sigma\rangle}\right) .
$$

The index $a$, however, does not depend on the choice of a generator of $\langle\sigma\rangle$ according to $(2)$, so one has

$$
a=\left(R: \sum_{j=0}^{m-1} R^{\left\langle\sigma^{j} v\right\rangle}+R^{\langle\sigma\rangle}\right)
$$

Using Proposition 14 of [7] we obtain

$$
a=\left(R: R_{1}\right)
$$

where $R_{1}=\langle\sigma\rangle^{\sim} R+\sum_{j=0}^{m-1}\left\langle\sigma^{j} \tau\right\rangle^{\sim} R$.

Since for any subgroup $H$ of $G, G^{\sim} Z[G]$ is an ideal of $H^{\sim} \boldsymbol{Z}[G]$, we have a $\boldsymbol{Z}$-isomorphism

$$
Z[G] / T \simeq R / R_{1},
$$


where $T=\langle\sigma\rangle^{\sim} \mathbb{Z}[G]+\sum_{j=0}^{m-1}\left\langle\sigma^{j} \tau\right\rangle^{\sim} \mathcal{Z}[G]$.

Now we need

LEMMA 1. Let $H<G$ and let $G=\bigcup_{i=1}^{N} H g_{i}$ be the decomposition of $G$ into the union of disjoint right cosets. Then

$$
H^{\sim} Z[G]=H^{\sim} \sum_{i=1}^{N} Z g_{i}
$$

Proof. Since for every $y \in Z[G]$

$$
y=\sum_{h \in H} \sum_{i=1}^{N} a_{h g_{i}} h g_{i},
$$

one obtains

$$
H^{\sim} y=\sum_{x \in H} \sum_{i=1}^{N} \sum_{w \in H} a_{h g_{i}} x h g_{i}=\sum_{x \in H} \sum_{i=1}^{N} \sum_{w \in H} a_{x-1} w_{g i} w g_{i}
$$

by substituting $w=x h$. Finally,

$$
H^{\sim} y=\sum_{w \in H} w \sum_{i=1}^{N}\left(\sum_{x \in H} a_{x-1_{w g}}\right) g_{i} .
$$

This lemma gives

$$
T=\langle\sigma\rangle \sim Z[\langle\tau\rangle]+\sum_{j=0}^{m-1}\left\langle\sigma^{j} \tau\right\rangle \sim Z[\langle\sigma\rangle]
$$

Now we shall use the formula

$$
a=(Z[G]: T)
$$

to estimate the index $a$. For convenience we shall assume that arithmetical operations on indices running from 0 to $p-1$ are performed $\bmod p$. If $x=\sum_{i=0}^{p-1} \sum_{j=0}^{m-1} \lambda_{i j} \tau^{j} \sigma^{i} \in Z[G]$ is an element of $T$, then there exist rational integers $a_{i j}$ and $\omega_{j}$ such that

$$
\left.x=\sum_{i=0}^{p-1} \sum_{j=0}^{m-1} \omega_{j} \tau^{j} \sigma^{i}+\sum_{s=0}^{m-1} \sum_{h=0}^{p-1} a_{h s} \sum_{j=0}^{m-1}\left(\sigma^{s} \tau\right)^{j}\right) \sigma^{h} .
$$

Applying the formula $\sigma^{i} \tau^{j}=\tau^{j} \sigma^{i r j}(r \bar{r} \equiv 1(\bmod p))$, we have

$$
\left(\sigma^{s} \tau\right)^{j}=\tau^{j} \sigma^{s \varphi_{j}}
$$

where $\varphi_{0}=0, \varphi_{j}=\bar{r}+\bar{r}^{2}+\ldots+\bar{r}^{\prime}$ with $1 \leqslant j \leqslant m-1$. Thus

$$
\begin{aligned}
x & =\sum_{s=0}^{m-1} \sum_{j=0}^{m-1} \sum_{h=0}^{p-1} a_{h s} \tau^{j} \sigma^{s \varphi_{j}+h}+\sum_{i=0}^{p-1} \sum_{j=0}^{m-1} \omega_{j} \tau^{j} \sigma^{i} \\
& =\sum_{i=0}^{p-1} \sum_{j=0}^{m-1}\left(\omega_{j}+\sum_{s=0}^{m-1} a_{i-s \varphi_{j}, s} \tau^{j} \sigma^{i}\right.
\end{aligned}
$$

by substituting $i=s \varphi_{j}+h$ in the first term. It follows that

$$
\lambda_{i j}=\omega_{j}+\sum_{s=0}^{m-1} a_{i-s \varphi_{j}, s}
$$

where $0 \leqslant i \leqslant p-1,0 \leqslant j \leqslant m-1$.

Let $\mu_{i j}=\lambda_{i j}-\lambda_{i 0}$ and let, for $1 \leqslant d \leqslant m-2$ and $d+1 \leqslant j \leqslant m-1, x_{h j}^{(d)}$ be integer solutions of the following system of linear congruences:

$$
\sum_{h=1}^{d} x_{h j}^{(d)} \varphi_{h}^{d-w}+\varphi_{j}^{d-w} \equiv 0(\bmod p)
$$

where $0 \leqslant w \leqslant d-1$. The system (5) is always solvable because $0, \varphi_{1}, \ldots, \varphi_{d}$ are distinct $\bmod p$.

Now we shall show that

$$
F_{j}^{(0)}=\sum_{i=0}^{p-1} \mu_{i j} \equiv 0(\bmod p)
$$

for $1 \leqslant j \leqslant m-1$ and

$$
F_{j}^{(d)}=\sum_{i=0}^{p-1} i^{d}\left(\mu_{i j}+\sum_{h=1}^{d} x_{h j}^{(d)} \mu_{i h}\right) \equiv 0(\bmod p)
$$

for $1 \leqslant d \leqslant m-2, d+1 \leqslant j \leqslant m-1$. The congruences (6) follow immediately from (4). To prove (7), we use (4) to get

$$
\begin{aligned}
F_{j}^{(d)}= & {\left[\omega_{j}-\omega_{0}+\sum_{h=1}^{d} x_{h j}^{(d)}\left(\omega_{h}-\omega_{0}\right)\right] \sum_{i=0}^{p-1} i^{d} } \\
& +\sum_{i=0}^{p-1} i^{d} \sum_{s=0}^{m-1} a_{i-s \varphi_{j, s} s}-\sum_{i=0}^{p-1} i^{d} \sum_{s=0}^{m-1} a_{i s} \\
& +\sum_{i=0}^{p-1} i^{d} \sum_{h=1}^{d} x_{h)}^{(d)} \sum_{s=0}^{m-1} a_{i-s \varphi_{h}, s}-\sum_{i=0}^{p-1} i^{d} \sum_{h=1}^{d} x_{h j}^{(d)} \sum_{s=0}^{m-1} a_{i s} .
\end{aligned}
$$

Since $\sum_{i=0}^{p-1} i^{d} \equiv 0(\bmod p)$ one obtains

$$
\begin{aligned}
F_{j}^{(d)} & \equiv \sum_{s=0}^{m-1} \sum_{i=0}^{n-1}\left\{\sum_{h=1}^{d} x_{h}^{(d)}\left[\left(i+s \varphi_{h}\right)^{d}-i^{d}\right]+\left[\left(i+s \varphi_{j}\right)^{d}-i^{d}\right]\right\} a_{i s} \\
& \equiv \sum_{s=0}^{m-1} \sum_{i=0}^{p-1} \sum_{w=0}^{d-1}\left(\begin{array}{l}
d \\
w
\end{array}\right) s^{d-w} i^{d}\left[\varphi_{j}^{d-w}+\sum_{h=1}^{d} x_{h j}^{(d)} \varphi_{h}^{d-w}\right] a_{i s} \equiv 0(\bmod p)
\end{aligned}
$$

by (5). 
Thus the coordinates of $x$ are zeros of the linear forms $F_{j}^{(d)}$ which turn out to be linearly independent $\bmod p$. To prove this suppose that there are integers $\alpha_{j}^{(d)}$ such that

$$
F=\sum_{j=1}^{m-1} \alpha_{j}^{(0)} F_{j}^{(0)}+\sum_{d=1}^{m-2} \sum_{j=d+1}^{m-1} \alpha_{j}^{(d)} F_{j}^{(d)}
$$

is a linear form with coefficients vanishing $\bmod p$.

Though $\alpha_{j}^{(d)}$ are defined only for $d+1 \leqslant j \leqslant m-1$ we extend their range by putting $\alpha_{j}^{(d)}=0$ for $1 \leqslant j \leqslant d$. We also put $x_{h i j}^{(d)}=0$ for $d+1 \leqslant h \leqslant m-1$.

Using (6) and (7), we get

$$
\begin{aligned}
F & =\sum_{j=1}^{m-1} \sum_{i=0}^{p-1} \alpha_{j}^{(0)} \mu_{i j}+\sum_{d=1}^{m-2} \sum_{j=d+1}^{m-1} \sum_{i=0}^{p-1} i^{d} \alpha_{j}^{(d)} \mu_{i j}+\sum_{i=0}^{p-1} \sum_{d=1}^{m-2} \sum_{j=d+1}^{m-1} \sum_{h=1}^{d} i^{d} \alpha_{j}^{(d)} x_{h j}^{(d)} \mu_{i h} \\
& =\sum_{i=0}^{p-1} \sum_{j=1}^{m-1} \sum_{d=0}^{m-2} i^{d} \alpha_{j}^{(d)} \mu_{i j}+\sum_{i=0}^{p-1} \sum_{h=1}^{m-1} \sum_{d=1}^{m-2} \sum_{j=d+1}^{m-1} i^{d} \alpha_{j}^{(d)} \mu_{i h} \\
& =\sum_{i=0}^{p-1} \sum_{j=1}^{m-1}\left[\alpha_{j}^{(0)}+\sum_{d=1}^{m-2}\left(\alpha_{j}^{(d)}+\sum_{i=d+1}^{m-1} \alpha_{t}^{(d)} x_{j t}^{(d)}\right) i^{d}\right] \mu_{i j}
\end{aligned}
$$

by substituting in the last but one line $j$ for $h$ and $t$ for $j$ in the second term. Since the coefficients of $F$ are all equal to $0(\bmod p)$ it follows that for $d+1 \leqslant j \leqslant m-1$

$$
\alpha_{j}^{(0)}+\sum_{d=1}^{m-2}\left(\alpha_{j}^{(d)}+\sum_{t=d+1}^{m-1} \alpha_{t}^{(d)} x_{j i}^{(d)}\right) i^{d} \equiv 0(\bmod p)
$$

But by definition $x_{j i}^{(d)}=0$ for $d+1 \leqslant j \leqslant m-1$, so

$$
\sum_{d=1}^{m-2} \alpha_{j}^{(d)} i^{d} \equiv 0(\bmod p)
$$

where $i=0, \ldots, p-1$. Thus for $d+1 \leqslant j \leqslant m-1$ we obtain

$$
\alpha_{j}^{(d)} \equiv 0(\bmod p)
$$

which proves the linear independence $\bmod p$ of the $m(m-1) / 2$ linear forms $F_{j}^{(d)}$.

Since by Théorème 10 of [3] the index $a$ is a power of $p$, (3) implies now

$$
a=p^{i w}
$$

where $w \geqslant m(m-1) / 2$. This together with (2) establishes Theorem 1 .

4. Now we shall prove three lemmas.

Lemma 2. Let $M_{0}, M_{1}, N$ be $Z[G]$-modules and

$$
f, f^{\prime} \in Z^{1}\left(G, \operatorname{Hom}_{\boldsymbol{z}}\left(M_{1}, N\right)\right), \quad f_{0} \in Z^{1}\left(G, \operatorname{Hom}_{\boldsymbol{z}}\left(M_{0}, N\right)\right) .
$$

If $\operatorname{Hom}_{\mathbf{Z}[G]}\left(N, M_{1}\right)=0$ and the extensions $\left(M_{1}, N ; f\right),\left(M_{1}, N ; f^{\prime}\right)$ are $Z[G]$-isomorphic, then there exists a $Z[G]$-isomorphism

$$
\left(M_{0} \oplus M_{1} \oplus M_{1}, N ; f_{0}, f, f^{\prime}\right) \simeq\left(M_{0} \oplus M_{1}, N ; f_{0} y, f\right) \oplus M_{1},
$$

where $v$ is a suitable automorphism of $N$.

Proof. Since $\operatorname{Hom}_{\mathrm{Z}[G]}\left(N, M_{1}\right)=0$, we can use Corollary (34.5) of [1]. Thus there exist $\lambda \in \operatorname{Aut}\left(M_{1}\right), v \in \operatorname{Aut}(N)$ and $c \in \operatorname{Hom}_{Z}\left(N, M_{1}\right)$ such that

$$
\lambda f_{g}^{\prime}(m)=f_{g}(v m)+g c(m)-c(g m), \quad g \in G
$$

and thus we can define a $Z[G]$-isomorphism

$$
\Psi:\left(M_{0} \oplus M_{1} \oplus M_{1}, N ; f_{0}, f, f^{\prime}\right) \rightarrow\left(M_{0} \oplus M_{1}, N ; f_{0} v, f\right) \oplus M_{1}
$$

by putting

$$
\left(m_{0}, m_{1}, m_{1}^{\prime}, n\right) \stackrel{\leftrightarrow}{\rightarrow}\left(m_{0}, 2 m_{1}-\lambda m_{1}^{\prime}-c(n), v \dot{n}, m_{1}-\lambda m_{1}^{\prime}-c(n)\right) .
$$

From now on we confine ourselves to the case where $m=q$ is a prime.

LEMma 3. If $\gamma$ is a $Z[G]$-generator for the $Z[G]$-module $Z[0]$, then

$$
\operatorname{An}(\gamma)=Z[G](1-\sigma)+Z[G]\langle\tau\rangle \sim
$$

where $\operatorname{An}(\gamma)$ denotes the annihilator ideal of $\gamma$.

Pro of. If $\alpha, \beta$ are $Z[G]$-generators of $Z[\theta]$, then they are units in the ring $\boldsymbol{Z}[0]$, so there is a $\boldsymbol{Z}[G]$-isomorphism $\varphi$ of $\boldsymbol{Z}[\theta]$ such that $\varphi(\alpha)=\beta$. Since $\operatorname{An}(\varphi(\alpha))=\operatorname{An}(\alpha)$ we may assume $\gamma=1$. Let

$$
x=\sum_{i=0}^{p-1} \sum_{j=0}^{q-1} a_{i j} \sigma^{i} \tau^{j} \in \mathbb{Z}[G]
$$

be an element of $\operatorname{An}(1)$. Then

$$
x \cdot 1=\sum_{i=0}^{q-1} \sum_{i=0}^{p-1} a_{i j} \theta^{j}=\sum_{j=0}^{q-2} \sum_{i=0}^{p-1}\left(a_{i j}-a_{i, q-1}\right) \theta^{j}=0
$$

so

$$
\sum_{i=0}^{p-1} a_{i j}=\sum_{i=0}^{p-1} a_{i, q-1}
$$

for $j=0, \ldots, q-1$. Therefore we have

$$
\begin{aligned}
x & =\sum_{i=0}^{p-1} \sum_{j=0}^{q-1} a_{i 0} \sigma^{i} \tau^{j}+\sum_{i=0}^{p-1} \sum_{j=0}^{q-1}\left(a_{i j}-a_{i 0}\right) \sigma^{i} \tau^{j} \\
& =\left(\sum_{i=0}^{p-1} a_{i 0} \sigma^{i}\right) \sum_{j=0}^{q-1} \tau^{j}+\sum_{j=0}^{q-1} \sum_{i=0}^{p-1}\left(a_{i j}-a_{i 0}\right)\left(\sigma^{i} \tau^{j}-\tau^{j}\right) \\
& \in Z[G]\langle\tau\rangle \sim+Z[G](1-\sigma) .
\end{aligned}
$$

Since the opposite inclusion is obvious, Lemma 3 is proved. 
LeMMA 4. If $e \geqslant 1$ and $\mathfrak{a}$ is an ideal of $A_{1}$ then the $Z[G]$-module $\mathbb{Z}[\theta] \oplus \mathscr{P e} \mathfrak{a}$ is not cyclic.

Proof. Assume to the contrary that $\mathbb{Z}[\theta] \oplus \mathscr{P P} \mathfrak{a}$ is cyclic with a generator $\left(\gamma,(1-\zeta)^{e} u\right)$. Then, by Lemme 1(i) of [8], $\gamma$ is a generator of $\mathbb{Z}[\theta]$ and $(1-\zeta)^{e} u$ is a generator of $\mathscr{P P}^{\mathfrak{e}} \mathfrak{a}$.

If we put $\delta=\sum_{j=0}^{q-1} \psi^{j}\left[(1-\zeta)^{e} u\right]$, then $\delta \in A_{1}$, and $\delta \in \mathscr{P}_{\mathfrak{a}}$ since $e \geqslant 1$. It follows that $\delta \in \mathscr{P}_{1} \mathfrak{a}$; but $\mathscr{P}_{1}$ totally ramifies in $Q(\zeta)$ and so $\delta \in \mathscr{P} 4 \mathfrak{a}$. Now applying the identity

$$
Z[G] x=\sum_{j=0}^{q-1} A \psi^{j}(x)
$$

$x \in \mathscr{P P}^{\mathfrak{a}}$, we obtain

$$
Z[G]\langle\tau\rangle \sim(1-\zeta)^{e} u=Z[G] \cdot \delta=A \delta \subset \mathscr{P}^{a} \mathfrak{a} \subset \mathscr{P}^{e+1} \mathfrak{a}
$$

and

$$
\boldsymbol{Z}[G](1-\sigma)(1-\zeta)^{e} u=Z[G](1-\zeta)^{e+1} u \subset \mathscr{P P}^{e+1} \mathfrak{a}
$$

because $1 \leqslant e \leqslant q-1$. By Lemma 3

$$
\operatorname{An}(\gamma)(1-\zeta)^{e} u \subset \mathscr{P}^{e+1} a .
$$

Lemme 1(ii) of [8] now implies that $\left(\gamma,(1-\zeta)^{e} u\right)$ cannot be a generator for $Z[\theta] \oplus \mathscr{P} e_{\mathfrak{a}}$, contrary to our assumption. This completes the proof of Lemma 4 .

Two $Z[G]$-modules $M$ and $M^{\prime}$ are said to be in the same genus if for every prime $l$, the $Z_{l}[G]$-modules $\boldsymbol{Z}_{(l)} \otimes M$ and $\boldsymbol{Z}_{(l)} \otimes M^{\prime}$ are $\boldsymbol{Z}_{(l)}[G]$-isomorphic, where $\boldsymbol{Z}_{(l)}$ is the localization of $\boldsymbol{Z}$ at $l$.

Now we are able to prove

THEOREM 2. Let $K$ be a real metacyclic extension of degree pq over $Q$, where $p, q$ are odd primes for which $q \mid p-1, q^{2} \nmid p-1$. Then:

(i) If $K$ has a Minkowski unit, then there exists an ideal $\mathrm{a} \triangleleft A_{1}$ and

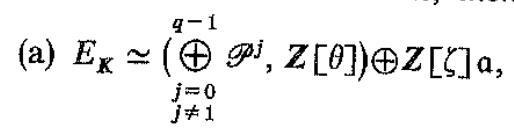

where all extensions $(\mathscr{P S}, Z[\theta])$ are non-trivial,

(b) $\frac{h_{k} h_{L}^{q}}{h_{K}}=p^{q-1}$,

(c) $N_{K / k}\left(E_{K}\right)=E_{k}$,

(d) the field $k$ has a Minkowski unit.

(ii) Conditions (b), (c) of (i) are necessary and sufficient for $E_{K}$ to be in the same genus as $Z[G] / Z G^{\sim}$.

(iii) If the class number of the field $Q(\zeta)^{\langle\psi\rangle}$ is 1 , then (b), (c) and (d) are necessary and suffcient for $K$ to have a Minkowski unit.

Proof. According to (1)

$$
E_{K} \simeq\left(\bigoplus_{j=0}^{s} \mathscr{P} e^{e_{j}} \mathfrak{a}_{j}, \mathfrak{b}\right) \oplus \underset{j=s+1}{\oplus} \overbrace{}^{q-1} \mathfrak{p e}_{j}
$$

where the extensions $\left(\mathscr{P}^{c_{j}} \mathfrak{a}_{j}, \mathfrak{b}\right)$ are non-trivial. The $Z[\langle\tau\rangle]$-module $\mathfrak{b}$ is an ideal of $Z[0]$ and by Lemme 2(i) of [8] it must be cyclic; hence, by Proposition 4 of $[8]$ it is isomorphic to $\mathbb{Z}[0]$. Since, by Proposition 6 of [4], there is exactly one non-trivial extension $\left(\mathscr{P P}^{e}, Z[\theta]\right)\left(e_{j} \neq 1\right)$, we may apply Lemma 2 to deduce that the $e_{j}$ 's are distinct for $0 \leqslant j \leqslant s$. Furthermore, Proposition 6 of [8] and the assumption of the cyclicity of $E_{K}$ yields that, for $s+1 \leqslant j \leqslant q-1$, the $e_{j}$ 's are also distinct. Since there is no non-trivial extension of $\mathscr{P}$ by $Z[\theta]$
(see (3) in [4]), we obtain

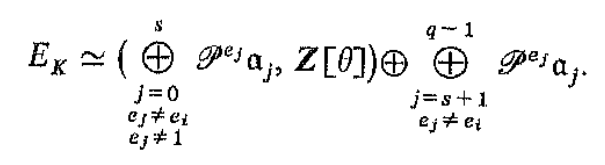

Using Lemma 4 and Lemme 3 of [8] we get $s=q-2$ and $e_{q-1}=0$. Thus finally we obtain (i) (a) because $E_{K}$ is determined up to isomorphism by the ideal class of the ideal $\prod_{j=0}^{q-1} a_{j}=a$. Now, (b) is a consequence of (a), of Proposition 2.4 in [6] and of (2), and (c) results from Corollaire of [7].

Since $Z[G] / Z G^{\sim}$ is cyclic and has $Z$-rank $p q-1$, we can write, by the same reasons as for $E_{K}$,

$$
Z[G] / Z G^{\sim} \simeq(\overbrace{\substack{j=0 \\ j \neq 1}}^{q-1} \mathscr{P} j, Z[\theta]) \oplus Z[\zeta] \mathfrak{a}^{\prime},
$$

where we may assume $\left(a_{j}^{\prime}, p q\right)=1$. Suppose that $E_{\mathrm{K}}$ and $\boldsymbol{Z}[G] / Z G^{\sim}$ are in the same genus. In particular, for $l \in\{p, q\}, Z_{(l)} \oplus E_{K}$ is $Z_{(l)}[G]$-cyclic and if we assume (1), we have

$$
Z_{(l)} \otimes E_{K} \simeq\left(\oplus_{j=0}^{q-1} \mathscr{P}_{l}^{e_{j}}, \boldsymbol{Z}_{(l)}[\theta]\right)
$$

where $\widetilde{\mathscr{P}}_{l}=Z_{(l)}[\zeta](1-\zeta)$. Since the above module is $Z_{(l)}[G]$-cyclic, since $(\mathscr{P} e, Z[\theta])$ is non-trivial if and only if $\left(\widetilde{\mathscr{P}}_{l}^{e}, Z_{(l)}[\theta]\right)$ is so for $l \in\{p, q\}$ (see (25.15) in [1]), and since all lemmas and propositions used in the proof of (i) (a) are also valid for $\boldsymbol{Z}_{(l)}[G]$-modules, we can proceed as above to obtain (i) (a). Owing to Proposition 2.3 and 2.4 of [6], this gives (b) and (c). 
Now suppose that (b) and (c) hold. Then Proposition 2.3 of [6] and (c) give

$$
E_{K} \simeq\left(\bigoplus_{\substack{j=0 \\ j \neq 1}}^{q-1} \mathscr{P P}^{j}, \mathfrak{b}\right) \oplus \mathscr{P P}^{e} \mathfrak{a},
$$

where $0 \leqslant e \leqslant q-1$ and $(\mathfrak{a}, p q)=1$. Using Proposition 2.4 of [6] and (b) together with (2) one has $e=0$. This proves (ii) because it suffices to localize at the primes dividing $G$ to show that $E_{\mathrm{K}}$ and $Z[G] / Z G^{\sim}$ are in the same genus.

To prove (iii) we need only show that conditions (b), (c) and (d) are sufficient. The condition for $p$ in (iii) shows that (a) and (b) imply

$$
E_{K} \simeq\left(\underset{\substack{q-1 \\ j \neq 0}}{\substack{q-1 \\ j \neq 1}} \mathscr{P}^{j}, \mathfrak{b}\right) \oplus Z[\zeta]
$$

According to the proof of Proposition 2.3 of $[6], N_{K / k}\left(E_{K}\right)$ and $p \mathfrak{b}$ are $\boldsymbol{Z}[G]$-isomorphic, so conditions (c) and (d) imply that $\mathrm{b}$ is $Z[G]$-cyclic, i.e., $\mathfrak{b} \simeq Z[\theta]$. Since there is exactly one, up to isomorphism, $Z[G]$-cyclic module of $\boldsymbol{Z}$-rank $p q-1$, namely $\boldsymbol{Z}[G] / Z G^{\sim}$, and this module is isomorphic to that in (8) with $b$ replaced by $Z[\theta]$, we conclude that $E_{K}$ is $Z[G]$-cyclic. Thus we have shown Theorem 2 .

Remark 1. Condition (i) (a) of Theorem 2 corrects a mistake in Lemme VI.5 of [5] and in Lemme 4.4 of [6]. The proofs of these lemmas base on the fact that every element of $Z[G] / Z G^{\sim}$ has a representative whose sum of its coefficients is zero, which is not true.

Remark 2. In (iii) of Theorem 2 condition (d) can be dropped if we assume that $q \leqslant 19$.

\section{References}

[1] C. W. Curtis and I. Reiner, Methods of representation theory, Wiley-Interscience, Now York 1981.

[2] D. Duval, Sur la structure galoisienne du groupe des unités d'un corps abélien récl de type $(p, p)$, J. Number Theory 13 (1981), 228-245.

[3] F. Halter-Koch and N. Moser, Sur le nombre de classes de certaines extensions métacycliques sur $Q$ ou sur un corps quadratique imaginaire, J. Math. Japan 30 (1978), 237.248.

[4] Len a Chang $\mathrm{Pu}$, Integral representation of non abelian groups of order $p q$, Michigan Math. J. $12(1965), 231-246$.

[5] N. Moser, Constraintes galoisiennes sur le groupe des unités de certaines extensions de $Q$ - applications arithmétiques, Thése, Grenoble, 1978.

[6] - Sur les unités d'une extension galoisienne non abélienne de degré pq du corps des rationnels, $p$ et $q$ nombres premiers impairs, Ann. Inst. Fourier (Grenoble) 291 (1979), 137-158.

[7] - Unités et nombre de classes d'une extension Galoisienne diédrale de Q, Abh. Math. Sem. Univer. Hamburg 48 (1979), 54-75.

[8] N. Moser, Théorème de densité de Tchebotareff et monogénéité de modules sur l'algèbre d'un groupe métacyclique, Acta Arith. 42 (1983), 303-323.

INSTITUTE OF MATHEMATICS

WROCLAW UNIVERSITY

Plac Grunwaldzki $2 / 4$

Received on 4.3 .1987

and in revised form on 22.6 .1987 